\title{
EVALUATION OF INTERACTION FORCES BETWEEN SURFACES IN ELECTROLYTE SOLUTIONS BY ATOMIC FORCE MICROSCOPE
}

\author{
Ko HIGASHITANI AND KaZUSHIGE ISHIMURA \\ Department of Chemical Engineering, Kyoto University, \\ Kyoto 606-01
}

Key Words: AFM Interaction Forces, Hydrated Ion, Adsorbed Layer, Hydration Force

\begin{abstract}
Interaction forces between a cleaved mica and the $\mathrm{SiO}_{2}$ surface of an apex of an $\mathrm{AFM}$ probe in electrolyte solutions were measured using an Atomic Force Microscope (AFM). It is found that (1) the AFM is a powerful apparatus to measure in-situ the interaction forces between surfaces in solutions on the molecular level, (2) the long-range interaction force between surfaces is estimated well by the classical DLVO theory, (3) the magnitude of the short-range interaction force corresponds to the order of the hydration enthalpy of the ions in the solution. A possible model for the structure of water molecules and ions adsorbed on the surface was discussed.
\end{abstract}

\section{Introduction}

Many materials, such as ceramics, pigments and magnetic tapes, form colloids in aqueous solutions during their production processes. The stability of colloids often influences the quality of the final product. Hence it is important to understand the detailed mechanism of the stability of colloids in aqueous solutions. Since stability is determined by the interaction forces acting between particle surfaces, the evaluation of interactive forces between solid surfaces is highly required not only to understand the fundamental mechanism of the interaction between surfaces, but also to control the properties of colloidal particles in industrial processes.

Interaction forces between solid surfaces in solutions have been studied extensively by Israelachvili (Israelachvili and Adams, 1976) and Pashley (Pashley, 1981a, 1981b, 1984, Pashley and Israelachvili, 1984a, 1984b) using a surface force apparatus (SFA), and the various characteristics of interactive forces have been clarified on the molecular level. The SFA is certainly a powerful apparatus to obtain in-situ information on interactive forces, but the surfaces employed must be smooth on the molecular level and transparent in principle. Because of these limitations for the surfaces, the SFA is not applicable to analyze the complicated surfaces of materials found in various industrial processes.

The atomic force microscope (AFM) (Binng and Quate et al., 1986) was invented in 1986. Using it, images of the unevenness of a surface are able to be evaluated with resolution of atomic order. It is found that the interactive forces between surfaces may be evaluated on the molecular level by the AFM.

* Received on May 20, 1996. Corrspondence concerning this article should be addressed to K. Higashitani.
The AFM has many advantages over the SFA in measuring interactive forces; (1) there is no limitation on the properties of surfaces examined, (2) local information of the surface properties is obtained, (3) the interactive force can be correlated with the image of the corresponding surface, and (4) handling of the apparatus is rather simple. These characteristics imply that the AFM is adequate to evaluate the interaction forces of surfaces of the various materials in industrial processes (Ducker and Senden, 1992, Karaman and Meagher etal., 1993, Larson and Drummond etal., 1993, Li and Tao etal., 1993, Senden and Drummond et al., 1994, Weisenhorn and Maivald et al., 1992).

In this study, interactive forces between a mica plate and a hemispherical $\mathrm{SiO}_{2}$ surface of the apex of an AFM probe in various electrolyte solutions were investigated to clarify the characteristics of the longrange interaction force due to the electrostatic double layer and the short-range interactive force due to water molecules and ions adsorbed on the surfaces, and a possible model for the structure of molecules adsorbed on the surface was proposed.

\section{Experiment}

\subsection{Medium}

Pure water of relative resistance $17.3 \mathrm{M} \Omega-\mathrm{cm}$ was prepared using reverse osmosis apparatus (Milli-Q, Millipore Co.). The electrolytes employed were a series of chlorides of reagent grade; chlorides of monovalent cations $\mathrm{LiCl}, \mathrm{NaCl}, \mathrm{KCl}$ and $\mathrm{CsCl}$ and chlorides of divalent cations $\mathrm{SrCl}_{2}, \mathrm{CaCl}_{2}$ and $\mathrm{MgCl}_{2}$. The electrolytes have different characteristics, as shown in Table 1, even though their valences are the same. Solutions were prepared dissolving the electrolyte into pure water and used within 6 hours to avoid contamination. The $\mathrm{pH}$ of the medium was 
Table 1 Characteristic of ions employed and thickness of adsorbed layer

\begin{tabular}{ccccc}
\hline Ion & $\begin{array}{c}\text { Diameter } \\
{[\mathrm{nm}]}\end{array}$ & $\begin{array}{c}\text { Hydration enthalpy } \\
{\left[\mathrm{kJ} \mathrm{mol}^{-1}\right]}\end{array}$ & $\begin{array}{c}\text { Hydrated ionic diameter } \\
{[\mathrm{nm}]}\end{array}$ & $\begin{array}{c}d / 2 \\
{[\mathrm{~nm}]}\end{array}$ \\
\hline $\mathrm{H}_{2} \mathrm{O}$ & 0.280 & - & - & 0.88 \\
\hline $\mathrm{Cs}^{+}$ & 0.338 & -301 & 0.66 & 0.40 \\
$\mathrm{~K}^{+}$ & 0.266 & -358 & 0.66 & 0.75 \\
$\mathrm{Na}^{+}$ & 0.190 & -443 & 0.72 & 0.85 \\
$\mathrm{Li}^{+}$ & 0.136 & -552 & 0.76 & 1.0 \\
\hline $\mathrm{Sr}^{2+}$ & 0.264 & -1519 & 0.82 & 0.75 \\
$\mathrm{Ca}^{2+}$ & 0.228 & -1666 & 0.82 & 1.0 \\
$\mathrm{Mg}^{2+}$ & 0.172 & -1996 & 0.86 & 1.5 \\
\hline
\end{tabular}

(Otaki, 1990, Israelachvili, 1985, Nightingale, 1959)

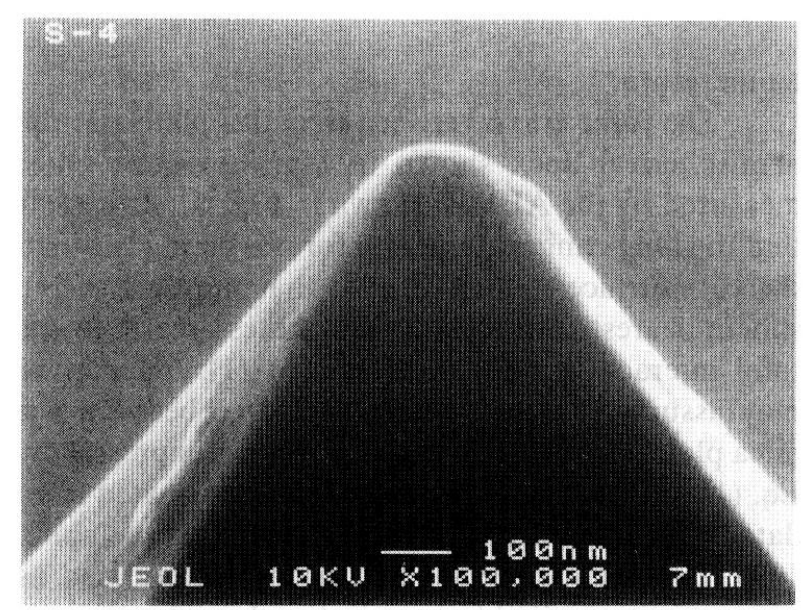

Fig. 1 SEM micrograph of $\mathrm{Si}_{3} \mathrm{~N}_{4}$ oxide probe coated by $\mathrm{SiO}_{2}$

$6.0 \pm 0.5$ in all the experiments.

\subsection{Surfaces}

In this study the surface of the mica plate and the $\mathrm{SiO}_{2}$ surface of the AFM probe were chosen as the test surfaces. Freshly cleaved mica was used as a flat surface in most cases, because the cleaved mica surface is known to be smooth on an atomic level. The mica surface usually bears a negative charge in aqueous solutions. When the dependence of the interaction force on the property of the flat surface was discussed, a plate of fused silica made from quartz glass was used as the flat surface.

The probes used were $\mathrm{Si}_{3} \mathrm{~N}_{4}$-oxide probes whose surface was coated by $\mathrm{SiO}_{2}$ and so negatively charged in the aqueous solution. The photo of the probe apex is shown in Fig. 1. It is clear that the apex surface may be regarded as hemispherical. Two different kinds of probes were used. When the longrange interaction force due to the electrostatic double layer was examined, the probes with the large apex (the radius of curvature $100<R<400 \mathrm{~nm}$ ) and the soft cantilever (the spring constant $k=0.12 \mathrm{nN} / \mathrm{m}$ ) were

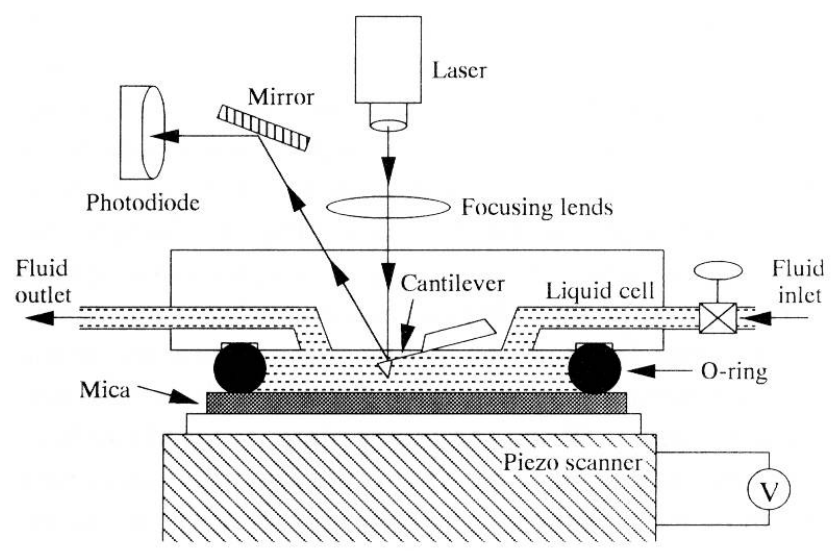

Fig. 2 Schematical drawing of AFM measuring system

employed. When the short-range interaction force due to the adsorbed layer on the surface was examined, the probes with the small apex $(R \sim 20 \mathrm{~nm})$ and the stiff cantilever $(k=0.38 \mathrm{nN} / \mathrm{m})$ were used to make the apex break through the adsorbed layer and contact fully with the mica surface.

\subsection{Measuring apparatus}

The AFM used was a Nanoscope-IIID (Digital Instruments) driven by Ver. 3.0 software under which a constant-speed single movement of specimen is possible. The measuring principle is briefly described here.

The measuring system is illustrated schematically in Fig. 2. After a mica plate was glued on the lower plate on the piezoelectric crystal, the mica and a probe were settled in a liquid cell with an O-ring which allows infinitesimal movement of the lower plate without leakage of the solution inside. The cell was filled with a given solution after rinsing its inside surface, and it was left standing for about 20 minutes until the movement of solution settled completely.

The lower plate was moved vertically with a constant speed by controlling the voltage to the piezoelectric system. The bend of the cantilever was 


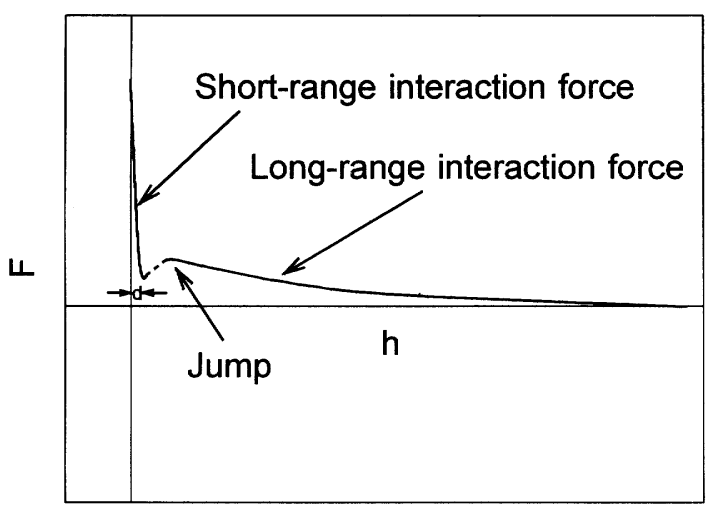

Fig. 3 Force curve between surfaces

monitored by the change of voltage from the split photodiode onto which was focused a laser beam reflected from the rear of the cantilever, and then the interaction force $F$ and the separation distance h between surfaces were calculated. A typical force curve obtained is illustrated in Fig. 3. When the mica approaches the probe apex, electrostatic repulsive force appears. When the two surfaces approach each other further, the probe jumps onto the mica surface because of attractive forces, such as van der Waals force. When the lower plate is raised further, the large repulsive force due to the adsorbed layer will be detected. When the probe moves with the same speed as the lower plate, the apex is considered to contact fully with the mica surface. The measurements were carried out at room temperature of $25 \pm 2{ }^{\circ} \mathrm{C}$.

\section{Results and Discussion}

\subsection{Long-range interaction force}

It is known that the long-range interaction force, which is attributable to the electrostatic repulsion and van der Waals attraction, acts between charged surfaces in aqueous solutions. According to the classical DLVO theory, the total long-range interaction force $F$ between a plate and a sphere of radius $R$ at the separation distance $\mathrm{h}$ is expressed by (Israelachvili, 1985).

$$
F=4 \pi \varepsilon_{0} \varepsilon R \psi_{1} \psi_{2} \kappa \exp (-\kappa h)-A R / 6 h^{2}
$$

where $\varepsilon_{0}$ and $\varepsilon$ are the relative permittivities of free space and the solution respectively, $\psi_{1}$ and $\psi_{2}$ are the apparent surface potentials of the sphere and plate, respectively, and $A$ is the Hamaker constant. $\kappa$, a measure of the ionic concentration of the medium, which is given by $\left(2 n_{0} Z^{2} e^{2} / \varepsilon_{0} \varepsilon k_{\mathrm{B}} T\right)^{1 / 2}$ (Israelachvili, 1985), where $n_{0}$ is the number concentration of ions, $Z$ is the valency of ions, $e$ is the elementary charge of electron, $k_{\mathrm{B}}$ is the Boltzmann constant and $T$ is the

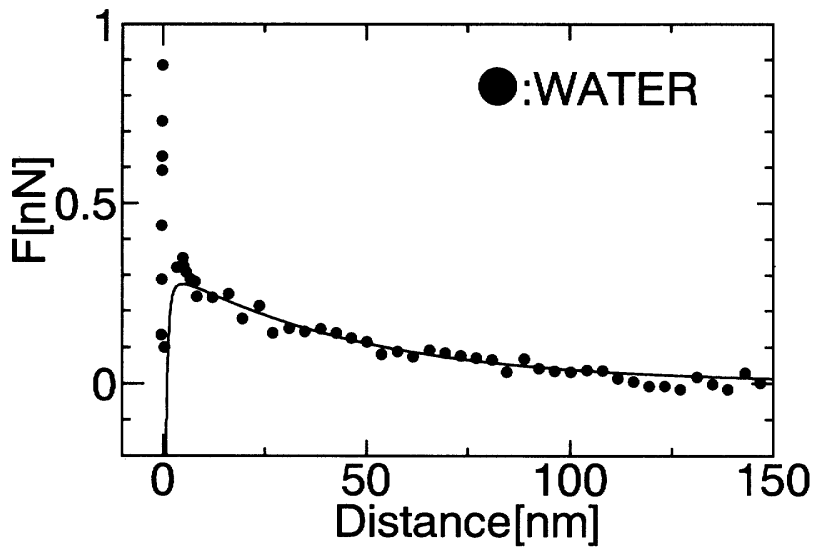

Fig. 4 Long-range interaction force in water: $R=130 \mathrm{~nm}$

temperature.

The force curve between the mica plate and the hemispherical apex shown in Fig. 1 in pure water measured by the AFM is shown in Fig. 4. It is clear that the long-range interaction force is acting between the two surfaces. It is known that the part of the sphere nearest to the plate contributes greatly to the total interaction force given by Eq. (1). Hence we may assume that the interaction force between the mica plate and the hemispherical apex is expressed by Eq. (1). The values of $\varepsilon_{0} \varepsilon$ and $A$ were estimated from data in the literature (The Chemical Society of Japan, 1993, Israelachvili, 1985) and $R$ was determined as $130 \mathrm{~nm}$ from the SEM photo in Fig. 1. Then Eq. (1) with two fitting parameters, $\left(\psi_{1} \psi_{2}\right)$ and $\kappa$, was fitted with the data as shown in Fig. 4. It is clear that the data are well expressed by Eq. (1). Then the values of $\left(\psi_{1} \psi_{2}\right)^{1 / 2}$ and $\kappa$ are determined to be $110 \mathrm{mV}$ and $0.021 \mathrm{~nm}^{-1}$, respectively. The magnitude of the averaged surface potential $\left(\psi_{1} \psi_{2}\right)^{1 / 2}$ agrees well with those of the mica and silica surfaces in water in the literature (Chapel, 1994, Hunter, 1981, Israelachvili, 1977, Lyons and Furlong et al., 1981, Pashley, 1981). The value of $\kappa$ corresponds to the ionic concentration of $4.1 \times 10^{-5} \mathrm{~mol} / 1$ of monovalent ions. We do not know the origin of this contamination, but salts are possibly dissolved from the mica plate.

Similar experiments were repeated in the various electrolyte solutions, and it was confirmed that the force curves were well fitted by Eq. (1). The determined values of $\kappa$ are shown in Table 2 comparing with those calculated from the amount of dosage of electrolytes. It is clear that the values of $\kappa$ agree reasonably well with the values expected from the dosage amount, irrespective of the kind of cations. The values of $\left(\psi_{1} \psi_{2}\right)$ determined by the fitting procedure were normalized by $\left(\psi_{10} \psi_{20}\right)$ for pure water and plotted against the electrolyte concentration $C_{\mathrm{e}}$ as shown in Fig. 5. It is clear that the magnitude of $\left(\psi_{1} \psi_{2}\right) /\left(\psi_{10} \psi_{20}\right)$ gradually decreases with increasing 
Table 2 Comparison of $\kappa$ between values calculated from dosage and estimated by Eq. (1)

\begin{tabular}{|c|c|c|c|c|c|}
\hline \multicolumn{3}{|c|}{ Electrolyte concentration $[\mathrm{mol} / \mathrm{l}]$} & $10^{-4}$ & $10^{-3}$ & $10^{-2}$ \\
\hline \multirow{5}{*}{$\kappa\left[\mathrm{nm}^{-1}\right]$} & \multicolumn{2}{|c|}{ Values calculated from dosage } & 0.039 & 0.11 & 0.33 \\
\hline & \multirow[t]{4}{*}{ Values estimated by Eq. (1) } & $\mathrm{CsCl}$ & 0.034 & 0.10 & 0.21 \\
\hline & & $\mathrm{KCl}$ & 0.040 & 0.11 & 0.28 \\
\hline & & $\mathrm{NaCl}$ & 0.042 & 0.095 & 0.31 \\
\hline & & $\mathrm{LiCl}$ & 0.045 & 0.10 & 0.26 \\
\hline
\end{tabular}

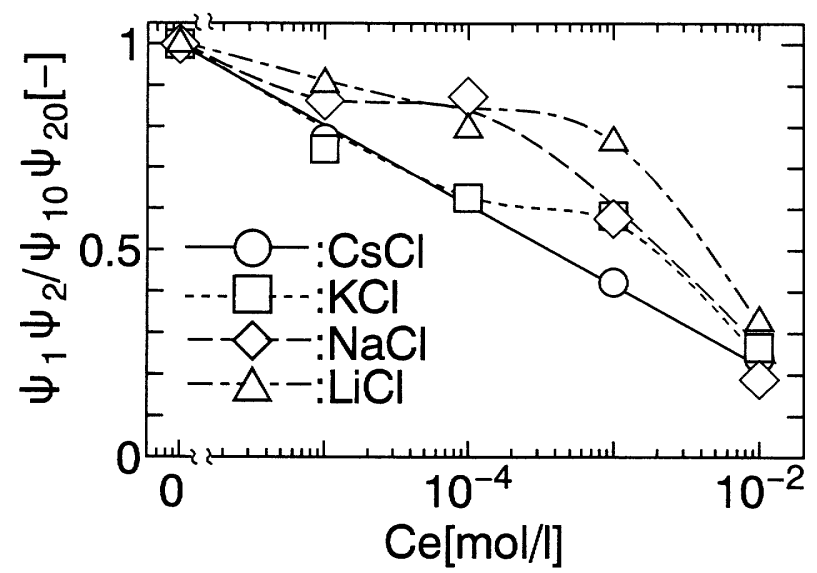

Fig. 5 Dependence of reduced potential on the electrolyte concentration

$C_{\mathrm{e}}$. This indicates that cations do adsorb on to the solid surface and the number of adsorbed cations increases with $C_{\mathrm{e}}$. It is interesting to note that the smaller the hydration enthalpy of cation is, the more rapidly the average surface potential decreases with $C_{\mathrm{e}}$. This seems to imply that cations of smaller hydration enthalpy adsorb on the surface more efficiently.

\subsection{Short-range interaction force in pure water}

The interaction force between a mica plate and a hemispherical $\mathrm{SiO}_{2}$ surface in pure water was measured as shown in Fig. 6, using the probe with the apex of $R \sim 20 \mathrm{~nm}$. A small long-range force appears at $h>3.2 \mathrm{~nm}$. This interaction force can be estimated by Eq. (1) using the data of $\left(\psi_{1} \psi_{2}\right)^{1 / 2}=110 \mathrm{mV}$ and $\kappa=0.021 \mathrm{~nm}^{-1}$ obtained in the previous experiment. It is clear that the prediction agrees well with the data and the effect of the long-range force is regarded negligibly small. At $h \sim 2.4 \mathrm{~nm}$, the probe jumped on the mica surface, and a very strong repulsive force appeared at $h<1.7 \mathrm{~nm}$. It is plausible to assume that this separation distance is a measure of the thickness of the adsorbed layer $d$, if the effect of the hydrodynamic interaction is negligible. It is known that the hydrodynamic interaction acts between a sphere and a plate when the sphere approaches to the surface at a high velocity. Figure 7 shows the dependence of $d$ on the approaching velocity of the mica plate $V_{\mathrm{a}}$. It is clear that the apparent value of $d$ is large when the

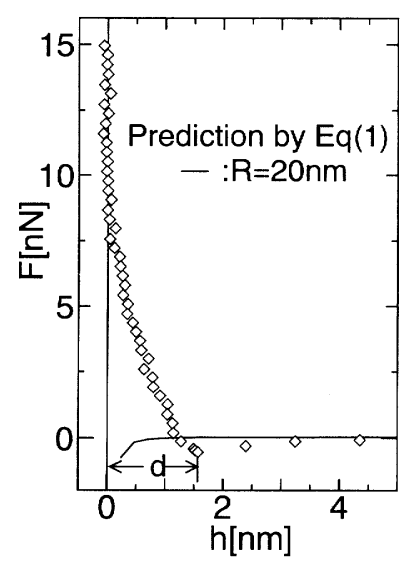

Fig. 6 Short-range interaction force in pure water

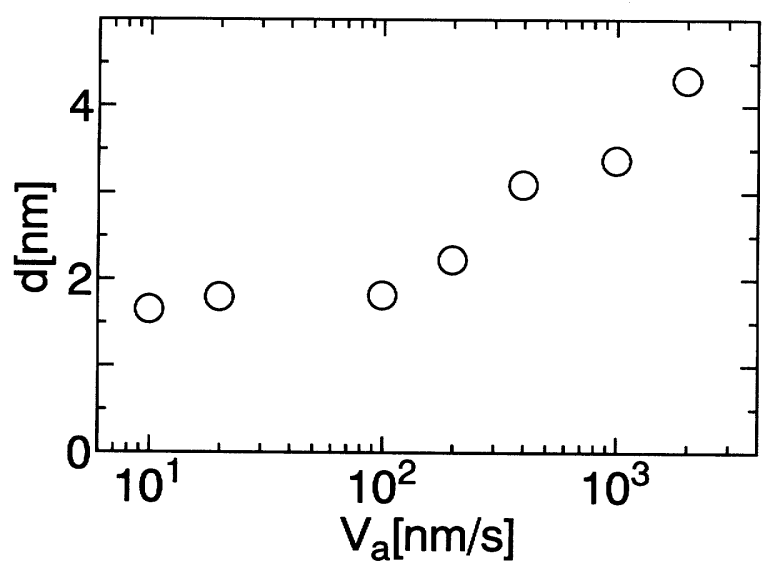

Fig. 7 Dependence of apparent adsorbed thickness on the approching velocity of surfaces: $R=20 \mathrm{~nm}$

plate velocity is very high, but the value of $d$ becomes constant at $V_{\mathrm{a}}<100 \mathrm{~nm} / \mathrm{s}$. The approaching velocity of the plate was taken to be less than $100 \mathrm{~nm} / \mathrm{s}$ in all the measurements.

According to the data of the short-range interaction force measured by the SFA (Chapel, 1994a, 1994b, Grabbe and Horn, 1993, "General Discussion," 1978, Pashley, 1981a, Peschel and Belouschek et al., 1982), no short-range force was detected between mica surfaces, although the force was observed between silica surfaces. In order to examine whether this is the case in our system, the force curve given in Fig. 6 


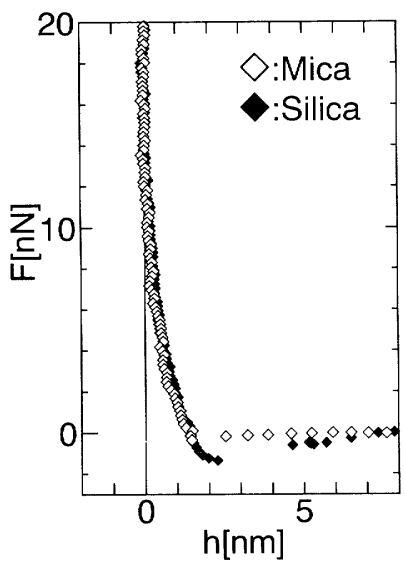

Fig. 8 Comparison of short-range force curve between mica and silica plates in water

was compared with that between a fused silica plate and the apex of AFM probe in pure water in Fig. 8. It is found that the force curves coincide with each other very well. Hence we consider that an adsorbed layer exists on the surface of the mica employed in this study. Further detailed experiments are required in order to clarify the difference between our data and the data given by the SFA. In the present study, we consider that the average thickness of the adsorbed layer on the mica and apex surfaces is given by $d / 2$, that is, $0.85 \mathrm{~nm}$ in the case of pure water. This thickness corresponds to the thickness of three layers of water molecules on the surface.

\subsection{Short-range interaction force in electrolyte solutions}

The short-range force curves in various electrolyte solutions were examined. Figure 9 shows a series of force curves for various $\mathrm{NaCl}$ concentrations, comparing with the force curve for pure water. It is interesting to note that the force curves do not vary with the $\mathrm{NaCl}$ concentration and coincide with that for pure water, even though $\mathrm{Na}^{+}$ions are considered to adsorb on the surface as expected from the data in
Fig. 5. On the other hand, the thickness of the adsorbed layer decreases with increasing concentration of $\mathrm{CsCl}$ as shown in Fig. 10. This difference between $\mathrm{NaCl}$ and $\mathrm{CsCl}$ solutions will be attributable to the difference in the hydration enthalpy of the cations.

The force curves for $1 \mathrm{~mol} / \mathrm{l}$ electrolyte solutions of monovalent and divalent cations are shown in Figs. 11 and 12, respectively, and their averaged thickness of adsorbed layer $d / 2$ is summarized in Table 1. It is clear that the strength of force curve and the magnitude of $d / 2$ follow the order of magnitude of hydration enthalpy among the cations of the same valency. This order of the adsorbed layer thickness agrees well with the data given elsewhere (Pashley and Israelachvili, 1984a, Pashley, 1994), although the magnitude in the present study is smaller.

\subsection{A possible model of adsorbed layer}

The above results do not give us enough information to clarify the detailed structure of the adsorbed layer on the solid surface. But it is still important for further investigation to discuss a possible structure of the adsorbed layer.

It is known that water molecules in the neighborhood of solid surfaces are more ordered than in the bulk. Therefore the short-range interaction force in pure water is attributable to the ordered water layer on the solid surface, whose thickness is three layers of water molecules.

In the case of $\mathrm{Cs}^{+}$ion, the magnitude of $d / 2$ decreases with increasing concentration as shown in Fig. 10 and $d / 2$ in Table 1 is smaller than that for the water layer and nearly equal to the diameter of bare ions. It is known that $\mathrm{Cs}^{+}$can not bind water molecules strongly enough because of the weak hydration force (Israelachvili, 1985). These features imply that $\mathrm{Cs}^{+}$adsorbs directly on the surface as shown in Fig. 13(a), and so the layer of adsorbed water will be destroyed. In the case of $\mathrm{Na}^{+}$ion, the value of $d / 2$ is nearly the same as that of water irrespective of

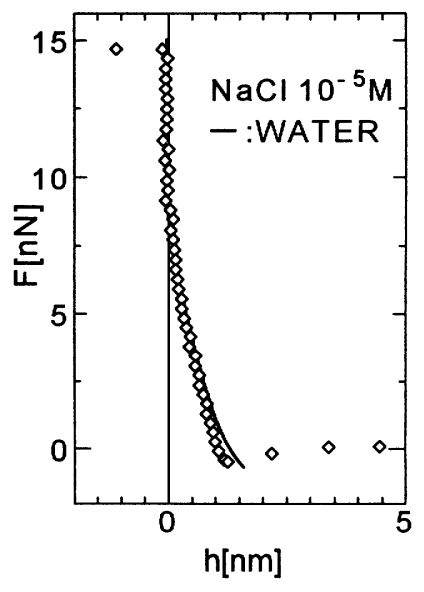

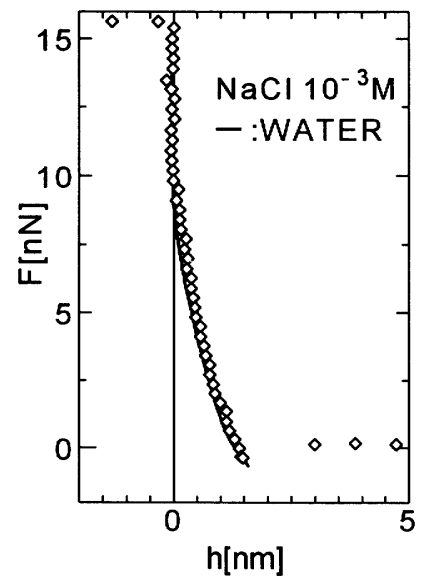
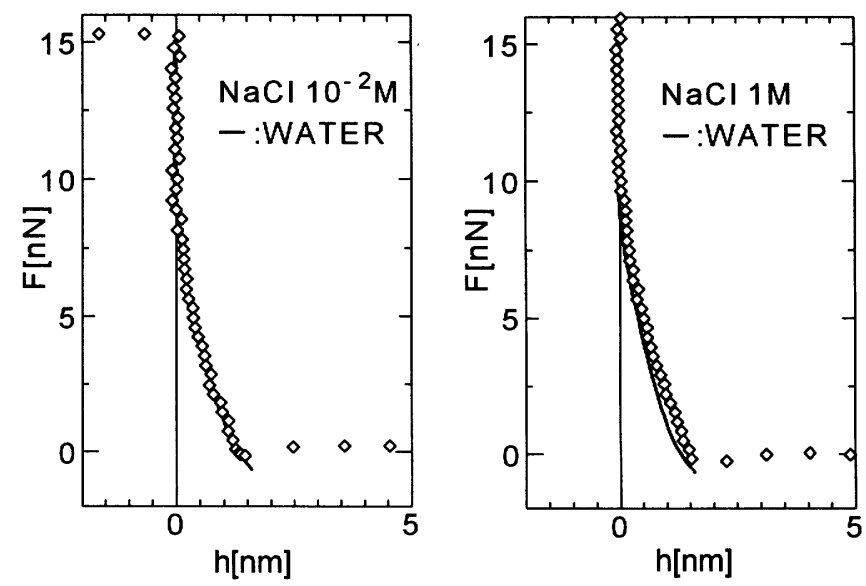

Fig. 9 Short-range interaction force in $\mathrm{NaCl}$ solution 

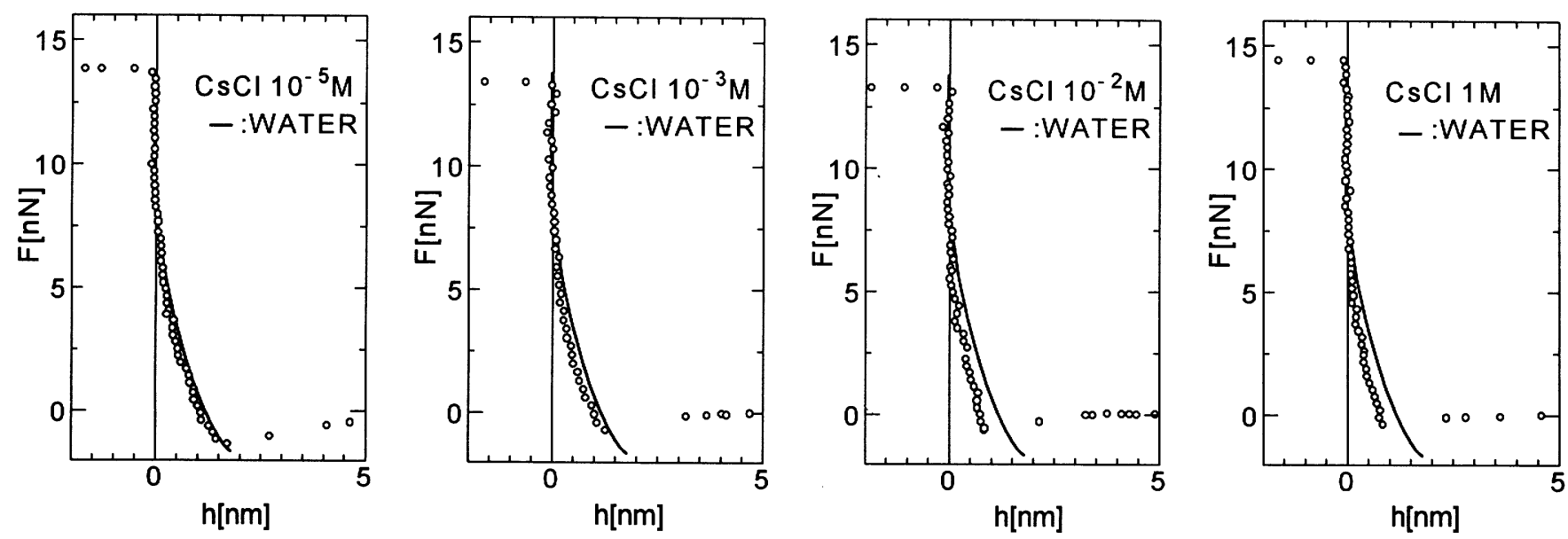

Fig. 10 Short-range interaction force in $\mathrm{CsCl}$ solution

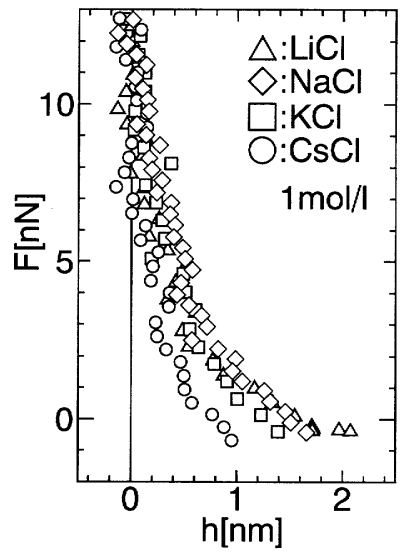

Fig. 11 Short-range interaction force in electrolyte solutions of monovalent cations

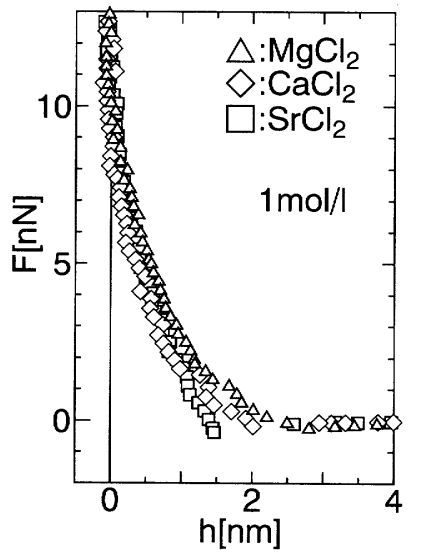

Fig. 12 Short-range interaction force in electrolyte solutions of divalent cations the concentration as shown in Fig. 9, and agrees approximately with the hydrated ionic diameters. These features imply that $\mathrm{Na}^{+}$ions with the bound water will migrate into the adsorbed layer of water molecules and a layer of hydrated $\mathrm{Na}^{+}$ions is formed as shown in Fig. 13(b). The values of $d / 2$ for $\mathrm{K}^{+}$, $\mathrm{Li}^{+}, \mathrm{Sr}^{2+}$ and $\mathrm{Ca}^{2+}$ are different corresponding with their hydrated ionic diameters, but their features are more or less the same as those for $\mathrm{Na}^{+}$. Hence we consider that the structure of the adsorbed layer of these ions may be illustrated by Fig. 13(b).

In the case of $\mathrm{Mg}^{2+}$ ion, the magnitude of $d / 2$ was found to be much greater than the size of the hydrated ions. The reason for this large value of $d / 2$ is not known at present, but it was suggested that $\mathrm{Mg}^{2+}$ near the surface reacts with water molecules and forms a complex (Pashley and Israelachvili, 1984a).

\section{Conclusion}

We investigated interaction forces between a mica plate and a $\mathrm{SiO}_{2}$ hemispherical surface of the apex of an AFM probe in electrolyte solutions and the following conclusions were drawn.

(1) The AFM is applicable to measure in-situ the interaction forces between surfaces in solutions on the molecular level.

(2) The interaction force between surfaces due to the electrostatic and van der Waals forces is estimated well by the classical DLVO theory.

(3) The short-range interaction force between the mica and $\mathrm{SiO}_{2}$ surfaces in pure water was observed.

(4) The magnitude of the short-range interaction force between surfaces in electrolyte solutions corresponds to the order of hydration enthalpy of the electrolyte.

(5) The following model for the adsorbed layer on the surface in solutions is suggested. About three layers of water molecules are adsorbed on surfaces in pure water. Ions of low hydration enthalpy, such as $\mathrm{Cs}^{+}$, absorb directly on the surface, but the ions of high hydration enthalpy, such as $\mathrm{K}^{+}, \mathrm{Na}^{+}, \mathrm{Li}^{+}, \mathrm{Sr}^{2+}$ and 


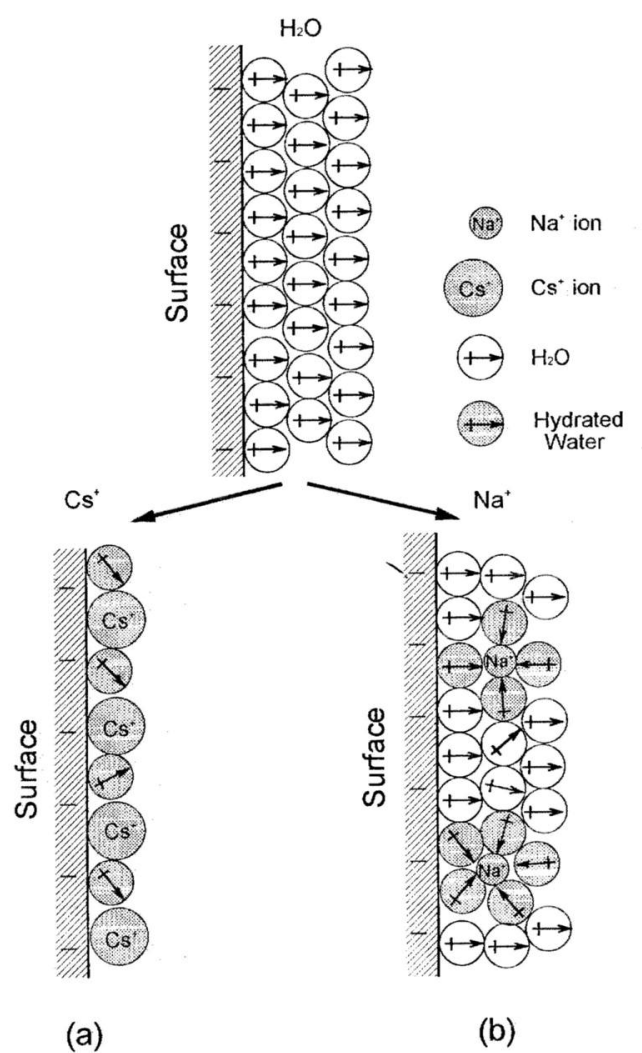

(a)

(b)

Fig. 13 Model of adsorbed layer

$\mathrm{Ca}^{2+}$, adsorb as the hydrated ions so that the thickness of the adsorbed layer is nearly equal to the size of hydrated ions. The ions which make the complex will form a much thicker adsorbed layer than the size of hydrated ion.

Nomenclature

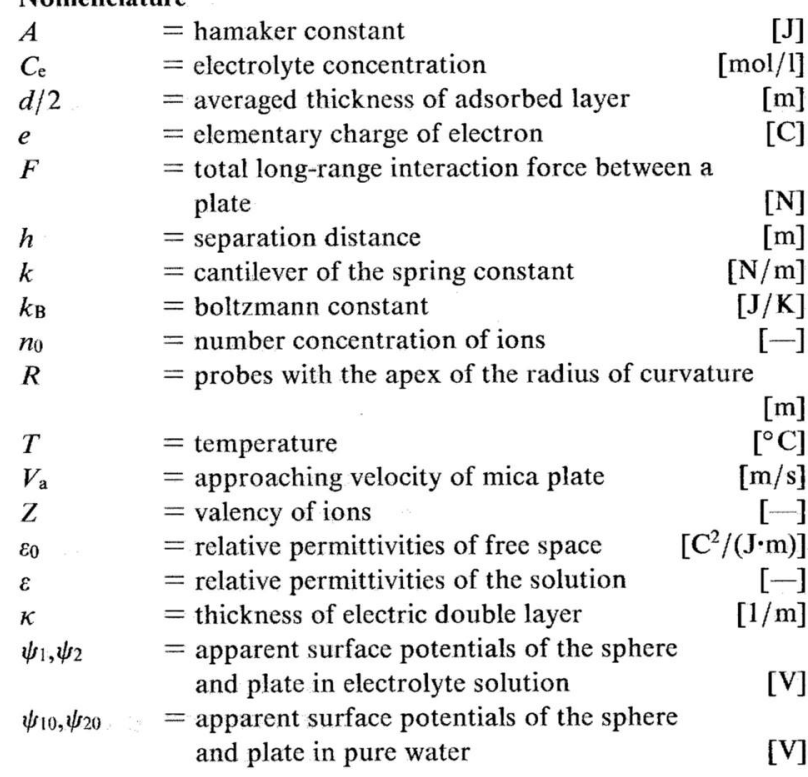

Literature Cited

Binng, G., C.F. Quate and Ch. Gerber; "Atomic Force Microscope," Phys. Rev. Lett., 56, 930 (1986)
Chapel, J.P.; "Electrolyte Species Dependent Hydration Forces between Silica Surfaces," Langmuir, 10, 4237 (1994a)

Chapel, J.-P.; "History-Dependent Hydration Forces Measured between Silica Surfaces," J. Colloid Interface Sci., 162, 517 (1994b)

The Chemical Society of Japan; Kagakubinran Kisohen II, 4th ed., p. 498, Maruzen, Tokyo, Japan (1993)

Ducker, W.A., T.J. Senden; "Measurement of Forces in Liquids Using a Force Microscope," Langmuir, 8, 1831 (1992)

"General Discussion," Faraday Discuss. Ceme. Soc., 65, 43 (1978)

Grabbe, A. and R.G. Horn; "Double-Layer and Hydration Forces Measured between Silica Sheets Subjected to Various Surface Treatments," J. Colloid Interface Sci., 157, 375 (1993)

Hunter, R.J.; Zeta Potential in Colloid Science, Academic Press, London, America (1981)

Israelachvili, J.N. and G.E. Adams; "Direct Measurement of Long Range Forces between Two Mica Surfaces in Aqueous $\mathrm{KNO}_{3}$ Solutions," Nature, 262, 774 (1976)

Israelachvili, J.N.; "Measurement of Forces between Surfaces Immersed in Electrolyte Solutions," Nature, 262, 20 (1977)

Israelachvili, J.N.; Intermolecular and Surface Forces, Academic Press, London, America (1985)

Karaman, M.E., L. Meagher and R.M. Pashely; "Surface Chemistry of Emulsion Polymerization," Langmuir, 9, 1220 (1993)

Larson, I., C.J. Drummond, D.Y.C. Chan and F. Grieser: "Direct Force Measurements between $\mathrm{TiO}_{2}$ Surfaces," J. Am. Chem. Soc., 115, 11885 (1993)

Li, Y.Q., N.J. Tao, J. Pan, A.A. Garcia and S.M.Lindsay; "Direct Measurement of Interaction Forces between Colloidal Particles Using the Scanning Forces Microscope," Langmuir, 9, 637 (1993)

Lyons, J.S., D.N. Furlong and T.W. Healy; “The Electrical DoubleLayer Properties of the Mica (Muscovite)-Aqueous Electrolyte Interface," Aust. J. Chem., 34, 1177 (1981)

Nightingale, E.R.; J. Phys. Chem., 63, 1381 (1959)

Ohtaki, H.; Ion no Suiwa, p. 30, Kyouritu Syuppan, Tokyo, Japan (1990)

Pashley, R.M.; "Hydration Forces between Mica Surfaces in Aqueous Electrolyte Solutions," J. Colloid Interface Sci., 80, 153 (1981a)

Pashley, R.M.; "DLVO and Hydration Forces between Mica Surfaces in $\mathrm{Li}^{+}, \mathrm{Na}^{+}, \mathrm{K}^{+}$, and $\mathrm{Cs}^{+}$Electrolyte Solutions: A Correlation of Double-layer and Hydration Forces with Surface Cation Exchange Properties," J. Colloid Interface Sci., 83, 531 (1981b)

Pashley, R.M.; "Hydration Forces between Mica Surfaces in Electrolyte Solutions," Adv. Colloid Interface Sci., 97, 446 (1984)

Pashley, R.M. and J.N. Israelachvili; "DLVO and Hydration Forces between Mica Surfaces in $\mathrm{Mg}^{2+}, \mathrm{Ca}^{2+}, \mathrm{Sr}^{2+}$, and $\mathrm{Ba}^{2+}$ Chloride Solutions," J. Colloid Interface Sci., 97, 446 (1984a)

Pashley, R.M. and J.N. Israelachvili; "Molecular Layering of Water in Thin Films between Mica Surfaces and Its Relation to Hydration Forces," J. Colloid Interface Sci., 101, 511 (1984b)

Peschel, G., P. Belouschek, M.M. Muller, M.R. Muller and R. Konig; "The Interaction of Solid Surfaces in Aqueous Systems," Colloid Polym. Sci., 260, 444 (1982)

Senden, T.J., C.J. Drummond and P. Kekicheff; "Atomic Force Microscopy: Imaging with Electrical Double Layer Interactions," Langmuir, 10, 358 (1994)

Weisenhorn, A.L., P. Maivald, H.-J. Butt and P.K. Hansma; "Measuring Adhesion, Attraction, and Repulsion between Surfaces in Liquids with an Atomic-Force Microscope," Phys. $\operatorname{Rev}$. (B), 45, 11226 (1992) 